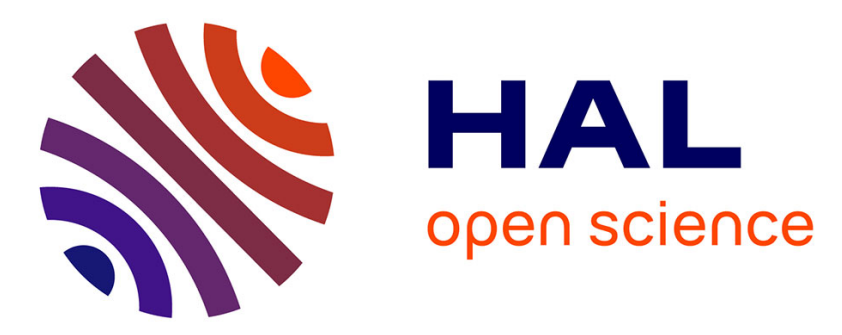

\title{
Le tournant environnemental de la société industrielle au prisme d'une histoire des débordements et de leurs conflits
}

\author{
Michel Letté
}

\section{- To cite this version:}

Michel Letté. Le tournant environnemental de la société industrielle au prisme d'une histoire des débordements et de leurs conflits. Vingtième siècle. Revue d'histoire, 2012, 113 (1), p. 142 à 154. halshs-00686274

\section{HAL Id: halshs-00686274 \\ https://shs.hal.science/halshs-00686274}

Submitted on 9 Apr 2012

HAL is a multi-disciplinary open access archive for the deposit and dissemination of scientific research documents, whether they are published or not. The documents may come from teaching and research institutions in France or abroad, or from public or private research centers.
L'archive ouverte pluridisciplinaire HAL, est destinée au dépôt et à la diffusion de documents scientifiques de niveau recherche, publiés ou non, émanant des établissements d'enseignement et de recherche français ou étrangers, des laboratoires publics ou privés. 


\title{
Le tournant environnemental de la société industrielle au prisme d'une histoire des débordements et de leurs conflits
}

\author{
Michel Letté
}

L'histoire environnementale évoque assez facilement l'idée d'un «tournant environnemental » qui, au cours des années 1960-1970, se serait traduit par une prise de conscience inédite. Une telle césure, suggère Michel Letté, est peut-être excessive. Une étude centrée sur les débordements industriels et leurs conflits, attentive aux contextes industriels et politiques, à l'entrelacs des acteurs, à leurs ressources protestataires, aux définitions du risque comme aux sensibilités au rapport entre nature et culture suggère un processus de beaucoup plus longue durée, dont les formes changent au cours du temps. C'est plutôt à un de ces déplacements que correspondent les années 1960-1970, dont il convient alors de repenser la particularité.

20 avril 2010, une plateforme pétrolière explose au large de la Nouvelle-Orléans. Elle sombre deux jours plus tard. Durant plusieurs mois, des centaines de milliers de litres de brut se déversent chaque jour dans le golfe du Mexique. Cet accident aux impacts sans précédent aura assurément renforcé le sentiment de démesure des débordements auxquels donne lieu aujourd'hui l'industrie. Il aura aussi accentué la pression qu'exerce l'opinion sur les pouvoirs publics pour exiger leur confinement.

Le confinement total est-il seulement possible ? La catastrophe de Fukushima en 2011 a ainsi rappelé que même dans le cas d'un dispositif où l'impératif d'une maîtrise sans faille est incontesté, les populations demeurent soumises au risque d'un débordement planétaire. Point culminant, avec Tchernobyl en 1986, d'une série d'accidents majeurs, Fukushima aura suscité un très fort regain d'intérêt pour l'histoire de l'expansion du nucléaire.

Il n'est cependant pas besoin que survienne l'accident pour que surgissent le conflit et l'histoire. Ainsi l'exploitation courante en mer des plates formes et la purge des réservoirs de pétroliers ont produit des volumes supérieurs de pollution à ceux des marées noires au cours des années 1960 et 1970, conduisant à de constantes protestations et à une régulation du transport maritime international. De même les centrales nucléaires débordent toujours le cadre strictement géographique de leur implantation, nécessitant de définir et donc de négocier par exemple le statut de leurs déchets, leurs traitements ou les seuils acceptables d'émission de 
leurs radiations. Le déversement régulier dans les cours d'eau, la pollution chronique et la dissémination de substances toxiques, la crainte de l'épidémie, la contamination des sols, l'imposition d'un risque non consenti, et bien d'autres débordements encore renvoient à une histoire industrielle comportant une bonne part de leurs contestations. Autrement dit, les débordements et leurs conflits ont largement contribué dans le passé à faire advenir l'exigence contemporaine de leur confinement. Elle n'est donc pas uniquement liée aux catastrophes les plus récentes, même si le sentiment pourrait bien prévaloir que les débordements ne seraient devenus inacceptables qu'avec le tournant environnemental de la société industrielle et l'invention de l'environnement au $20^{\mathrm{e}}$ siècle.

Bien avant l'industrialisation, les conflits aux abords des sites témoignent en réalité que le débordement est inhérent à de nombreuses activités humaines. Chroniques ou accidentels, les débordements provoquaient déjà la protestation des populations alentour. L'attention que leur portaient les autorités était cependant double. Elle était autant motivée par l'obsession de l'ordre public que par le souci de l'hygiène. S'était ensuite imposée au $19^{\mathrm{e}}$ siècle une autre exigence: encadrer les contestataires, afin de ne pas compromettre le développement de l'industrie $^{1}$. L'administration se dotait ainsi en 1810 d'une législation industrialiste opportune $^{2}$. C'est toutefois au travers d'un impératif de gestion des conflits que l'administration constituait en champ d'action politique la protection des voisins et des travailleurs $^{3}$. Les revendications environnementalistes au $20^{\mathrm{e}}$ siècle, comme l'implication croissante des riverains auront alors profondément transformé les cadres de l'intervention publique.

Dans quelle mesure les débordements et leurs conflits ont-ils contribué à ces évolutions ? Quelle a été leur influence sur le tournant environnemental de la société industrielle ? Ces interrogations sont aujourd'hui prégnantes parmi les gestionnaires de l'environnement. Elles

${ }^{1}$ ( ) Thomas Le Roux, Le Laboratoire des pollutions industrielles : Paris, 1770-1830, Paris, Albin Michel, 2011 ; id. et Jean-Baptiste Fressoz, «Protecting and Commodifying the Environment: The Great Transformation of French Pollution Regulation, 1700-1840 », in Geneviève Massard-Guilbaud et Stephen Mosley (dir.), Common Ground: Integrating the Social and Environmental in History, lieu ?, Cambridge Scholars Publishing, 2011, p. 340-366.

2 ( ) Geneviève Massard-Guilbaud, Histoire de la pollution industrielle : France, 1789-1914, Paris, Éd. de l'EHESS, 2010 ; Pierre Lascoumes, L'Éco-pouvoir : l'environnement entre nature et politique, Paris, La Découverte, 1994, p. 95-137.

${ }^{3}$ ( ) Gérard Jorland, Une société à soigner : hygiène et salubrité publiques en France au XIX siècle, Paris, Gallimard, 2010 ; Caroline Moriceau, Les Douleurs de l'industrie : l'hygiénisme industriel en France, 1860-1914, Paris, Éd. de l’EHESS, 2010. 
ont suscité un regain d'intérêt pour l'histoire des conflits associés aux activités polluantes et dangereuses. Celle-ci fait l'objet depuis d'importants travaux ${ }^{4}$. Ils assument de cette façon que l'histoire des conflits est aussi essentielle que celle de l'intervention publique qui leur succède. Cette dernière porte généralement sur le contrôle et la gestion du comportement des agents économiques et sociaux. Une perspective alternative est d'examiner ce qui a conduit à l'émergence au $20^{\mathrm{e}}$ siècle de l'environnement comme catégorie politique de la pensée et de l'action ${ }^{5}$. En effet, environnement et conflit sont constitutifs l'un de l'autre.

En suggérant une histoire des débordements eux-mêmes et des conflits auxquels ils ont donné lieu, l'environnement n'apparaît plus seulement comme l'émergence d'une conscience collective, mais comme la convergence de paradoxes à surmonter. Le tournant environnemental de la société industrielle coïncide en effet avec ce moment particulier où les impacts les plus visibles de l'activité industrielle s'effacent ou disparaissent. Les grandes usines polluantes tendent en France à se délocaliser. Les autres deviennent «propres » ou recourent aux progrès techniques incontestables pour minimiser leurs externalités. Dépollutions et réaffectations des sites anciens redonnent une couleur plus verdoyante aux quartiers durables. Pourtant l'industrie reste stigmatisée. Ses débordements n'ont plus droit de cité. Leur confinement est exigé. Le constat n'est cependant contradictoire qu'en apparence. En cherchant à réinscrire le tournant environnemental de la société industrielle dans le temps long, les conflits actuels deviennent au contraire le lieu d'une accumulation de débordements durant plus de deux siècles d'industrialisation. Leur histoire, à laquelle invite celle des catastrophes et des pollutions, est en réalité une histoire qui contient celle du tournant environnemental de la société industrielle et de l'invention de l'environnement au $20^{\mathrm{e}}$ siècle.

\section{De quoi « débordement » est-il le nom ?}

On s'intéresse donc ici à l'un des motifs du conflit environnemental : le débordement industriel. Pourquoi cependant recourir à cette notion plutôt qu'aux termes «pollution » et « nuisance »? Chercher à identifier des débordements permet d'abord de focaliser l'attention

${ }^{4}$ ( ) Jean-Baptiste Fressoz, " "La fin du monde par la science” : innovations, risques et régulations de l'inoculation à la machine à vapeur, 1750-1850 », thèse de doctorat, EHESS, 2009 ; Estelle Baret-Bourgoin, La Ville industrielle et ses poisons: les mutations des sensibilités aux nuisances et pollutions industrielles à Grenoble, 1810-1914, Grenoble, Presses universitaires de Grenoble, 2005 ; Christoph Bernardt et Geneviève Massard-Guilbaud (dir.), Le Démon moderne : la pollution dans les sociétés urbaines et industrielles d'Europe, Clermont-Ferrand, Presses Universitaires Blaise-Pascal, 2002.

5 ( ) Florian Charvolin, L'Invention de l'environnement en France : chroniques anthropologiques d'une institutionnalisation, Paris, La Découverte, 2003. 
sur le dispositif qui leur donne naissance. C'est de ce dernier qu'émane ce quelque chose engendrant la contestation. La proposition permet d'articuler trois registres d'analyse incontournables : le territoire, l'histoire, le caractère nécessairement hybride et pluriel des réalités dans lesquelles s'ancre le conflit.

Ensuite, la multitude et l'ambiguïté des phénomènes que recouvre la notion de débordement renvoient à une histoire non seulement des faits ou des conséquences tangibles, mais surtout des processus par lesquels ces derniers adviennent dans des espaces politiques et sociaux. Autrement dit, considérer ce qui déborde invite à restituer les traductions, qualifications et autres conventions visant à rendre réel ce qui, traversant les limites d'un dispositif de production, devient une justification de conflit. La démarche suppose de partir des contestations par lesquelles l'insertion d'un dispositif et ses extensions sont devenues des débordements. L'environnement peut être alors vu comme le théâtre de négociations entre des acteurs concernés ou affectés, qui tentent de faire exister ou de disparaître les débordements stigmatisés.

Enfin, le mot contient le problème. Ce qui déborde est soupçonné d'illégitimité, est perçu comme ce qui doit être contenu, contrôlé, surveillé, voire stoppé sous peine de catastrophe. Son statut sera toujours ambigu. Le débordement n'est pas nécessairement une matière tangible, mais il peut être immatériel. Ainsi faut-il compter avec tout ce qui est susceptible de générer ou d'entretenir le conflit entre un dispositif et son environnement : débordements physiques et liés aux sens (odeurs, miasmes, fumées, vapeurs, bruits, trépidations, poussières, vues intolérables ou immorales, effluents, etc.). Ces derniers constituent au sens propre les nuisances et les pollutions ; débordements potentiels et redoutés, c'est-à-dire les risques ou les dangers supposés, fantasmés ou avérés; débordements invisibles, inconnus ou non perceptibles (vecteurs de contamination, radiations, toxiques incolores et inodores, etc.); débordements différés dans le temps (effets cumulatifs de polluants, mais aussi réactions anticipées des populations, par exemple au stade de l'annonce d'un projet d'implantation); débordements enfin d'ordre économique et social (troubles de l'ordre public, concurrence pour l'usage de la ressource, effet de la présence d'une entreprise sur le comportement des propriétaires fonciers, emprise d'une activité sur l'organisation de la vie locale, etc.).

Une histoire des débordements et de leurs conflits consisterait dès lors à préciser ce qui devient un débordement, ce pourquoi et comment tel débordement était perçu, vécu comme tel, et contre lequel il était jugé nécessaire de lutter, ou au contraire de feindre l'ignorance ou d'en accepter les conséquences. Partant d'une autre catastrophe survenue en 2010 en Hongrie, on illustrera dans ce qui suit comment peut ainsi être réactivé en France et relu, au prisme des 
débordements, un conflit environnemental engagé pourtant il y a plus d'un siècle.

\section{Renégocier emprise territoriale et environnement}

Le déferlement spectaculaire en 2010 de boues toxiques dans un rayon de plusieurs dizaines de kilomètres carrés, suite à la rupture du réservoir de l'usine d'alumine d'Ajka en Hongrie, est sans conteste un bel exemple de débordement industriel. Ce dernier, dont l'impact local a été une catastrophe écologique et humaine majeure, n'a pas été sans incidence en France où une usine similaire s'est implantée à Gardanne en 1893.

Cette dernière usine avait été construite avant même que ne soit obtenue l'autorisation préfectorale préalable à l'installation des établissements chimiques affectant l'environnement immédiat ${ }^{6}$. En trois ans, l'étendue du site avait déjà doublé. Dès les premières années, usines et riverains, notamment agriculteurs, sont en conflit. Les motifs sont en partie les poussières rouges que le Mistral emporte depuis les réserves de bauxite et qu'il dépose aux alentours, sur les maisons, les potagers, les champs. Les usines sont par ailleurs très bruyantes, de jour comme de nuit. Enfin l'odeur désagréable de l'alumine calcinée sature l'atmosphère ambiante.

Les débordements permanents qui suscitent le plus de tensions sont toutefois les boues dites rouges, résidus principaux de la fabrication d'alumine. Pour en produire une tonne, deux de bauxite sont nécessaires, ainsi qu'une de soude, quatre de lignite et beaucoup d'eau. La bauxite est concassée, broyée, puis attaquée par une liqueur de soude dans un autoclave sous pression à température élevée. Après filtration, les résidus de bauxite forment les boues rouges contestées, ou tout au moins leur gestion territoriale.

Au démarrage de la production, ces résidus sont déposés entre l'usine et la gare, puis de l'autre côté de la route. Leur teneur en soude est élevée. Les boues sont une nuisance. Elles sont toxiques. Une négociation avec les Eaux et Forêts aboutit en 1902 à autoriser leur évacuation sur les hauteurs de la commune voisine de Bouc-Bel-Air. Pour ce faire, un transporteur aérien est mis en service en 1906. Soixante années plus tard, et après de nombreux conflits, les vallons pollués sont considérés comme saturés. L'une des solutions envisagées pour l'évacuation de ces résidus est leur conduite en mer. Entre menace de fermeture de l'usine si les autorités publiques ne l'autorisait pas et une franche opposition

\footnotetext{
${ }^{6}$ ( ) Philippe Mioche, L'Alumine à Gardanne de 1893 à nos jours : une traversée industrielle en Provence, Grenoble, Presses universitaires de Grenoble, 1994 ; id., «L'alumine à Gardanne, de la deuxième industrialisation à la fin des années soixante», in Ivan Grinberg et Florence Hachez-Leroy (dir.), Industrialisation et sociétés en Europe occidentale de la fin du XIX siècle à nos jours : l'âge de l'aluminium, Paris, Armand Colin, 1997, p. 85-94.
} 
locale, notamment menée par Alain Bombard, les établissements Ricard et les associations de pêcheurs, une canalisation de quarante-cinq kilomètres est construite de Gardanne à PortMiou, prolongée de huit kilomètres sous la mer. À partir de 1967, ses boues sont acheminées en partie par ce conduit pour être rejetées au large de Cassis. Le reste est stocké dans un bassin près de l'usine.

L'insertion territoriale de ce dispositif de production, celle de la légitimité des risques imposés aux populations locales et ses extensions sous la forme de pollutions ont, comme dans la plupart des cas similaires en France, suscités de vifs conflits avec l'environnement ${ }^{7}$. Ces derniers, qui n'ont jamais cessé au niveau local, ont resurgi sur le plan national à l'occasion de la catastrophe d'Ajka en 2010 entre industriels, autorités publiques, populations environnantes et groupes écologistes. L'événement a notamment réactivé les négociations auparavant atténuées pour qualifier ces boues émanant de l'usine, autrement dit pour faire exister et disparaître les débordements auxquels donne lieu l'usine de Gardanne. On a ainsi vu réapparaitre la question de la qualification de ces résidus, celle surtout de la légitimité des territoires de l'entreprise qui sont à la fois ceux du site de production et de stockage des matières toxiques et de ceux qu'elle s'approprie par le déversement dans les fonds marins. La contestation environnementale porte bien, dans ce cas, sur l'attribution des fonctions de ces extensions territoriales. Elles sont pour les uns une assimilation nécessaire des externalités de l'usine. Elles sont pour d'autres une appropriation illégitime d'un milieu à préserver, ou tout au moins d'un territoire à partager. Ces fragments de territoires contestés deviennent alors des débordements et le motif d'un conflit. L'enjeu reste pour les contestataires de les qualifier comme tels. Le dispositif formé par l'usine et le déversement de ses boues déborde.

Les débordements apparaissent donc ici comme une variante territoriale des motifs à l'origine de conflits d'implantation et d'usage. Une industrie, un atelier, un champ ou une mine d'exploitation ne sont jamais posés sur une terre inhabitée vierge de toute histoire. Ils interagissent en permanence avec ce qui les entoure, s'ancrent dans des cultures locales. Tout dispositif négocie plus ou moins explicitement la reconnaissance de zones de compétences, de sphères d'influences, pour user de droits sur des fractions de territoires proportionnels aux pouvoirs qu'il peut y exercer. Ses débordements sont ainsi regardés comme des espaces nécessairement hybrides où les fondements objectifs du territoire matérialisent des enjeux économiques, politiques et sociaux. Au prisme des débordements, cette histoire du conflit

7 ( ) Marie-Claire Loison et Anne Pezet, «L'entreprise verte et les boues rouges : les pratiques controversées de la responsabilité sociétale à l'usine d'alumine de Gardanne (1960-1966)», Entreprises \& 
environnemental renvoie ici aux négociations pour une cohabitation toujours précaire entre des territoires aux fonctions contradictoires. Il importe dès lors de ne pas perdre de vue qu'ils sont le fait d'acteurs ${ }^{8}$. Parmi eux, les contestataires sont l'objet d'une attention particulière, notamment ceux dont la voix n'a que peu ou difficilement porté dans les espaces traditionnels de la cité pour parvenir distinctement et sans ambiguïté.

\section{Débordements et conflits avant l'environnement}

Avant que l'environnement n'élève la crainte de la pollution et des risques industriels au rang d'une évidence contre laquelle il s'agirait de lutter, les populations semblaient majoritairement consentir à en subir les conséquences. L'inconscience environnementale caractériserait-elle pour autant les périodes antérieures? Aurait-elle accompagné le consentement généralisé, le désir d'une économie rationnelle par l'industrialisation, tant parmi les populations que parmi les élites et les décideurs ? La contestation aurait-elle été en somme marginale, inaudible, sans justification légitime, soit du fait de l'ignorance des impacts de l'industrie, soit parce que l'inéluctable était amplement partagé ?

Loin d'avoir été totalement négligé par ceux qui transformaient l'économie industrielle, l'environnement était administré, tout comme l'étaient les populations, pour les besoins de la production rationnelle. L'histoire des pollutions montre que leurs conséquences n'ont jamais été ignorées, ni par les riverains ou les ouvriers concernés, ni par les autorités. Les témoignages sont anciens ${ }^{9}$. Leurs motifs se diversifient au fil des siècles ${ }^{10}$. La métallurgie, l'extraction minière et l'exploitation forestière suscitaient les conflits les plus nombreux ${ }^{11}$. Les plaintes des urbains au Moyen Âge étaient constantes contre les abattoirs, les mégissiers, la fonte du suif ou le traitement des métaux. La volonté des édiles était réelle de sortir la ville de la fange et des émanations putrides ou corrosives de ces ateliers ${ }^{12}$. Si des paysans étaient en lutte dans la campagne lyonnaise au début du $18^{\mathrm{e}}$ siècle contre l'exploitant de mine

Histoire, 45, 2006, p. 97-115.

${ }^{8}$ ( ) Hervé Gumuchian et al., Les Acteurs, ces oubliés du territoire, Paris, Anthropos, 2003.

9 ( ) J. Donald Hughes, Pan's Travail: Environmental Problems of the Ancient Greeks and Romans, Baltimore, Johns Hopkins University Press, 1996 ; Peter Brimblecombe, The Big Smoke : A History of Air Pollution in London since Medieval Times, Londres, Routledge, 1987.

${ }^{10}$ ( ) Corinne Beck, Yves Luginbühl et Tatiana Muxart (dir.), Temps et espaces des crises de l'environnement, Paris, Quae, 2006.

${ }^{11}$ ( ) «Violences et Environnement», Cahier d'études, dirigé par Andrée Corvol, 2, 1992 ; Denis Woronoff (dir.), Forges et Forêts : recherches sur la consommation proto-industrielle de bois, Paris, Éd. de l'EHESS, 1990.

${ }^{12}$ ( ) Jean-Pierre Leguay, La Pollution au Moyen-Âge, lieu ?, Jean-Paul Gisserot, 1999. 
François Blumenstein, c'était à Paris une série continue de plaintes pour faire cesser ou éloigner de la ville les nuisances industrielles ${ }^{13}$. Au tournant des $18^{\mathrm{e}}$ et $19^{\mathrm{e}}$ siècles, les soudières Leblanc associées à la fabrication d'acide sulfurique étaient à l'origine d'une partie significative des conflits environnementaux et des procès, à Rouen, à Lille ou à Marseille ${ }^{14}$. L'impact de ces activités est aujourd'hui encore profondément inscrit dans le paysage ${ }^{15}$. Les manifestations de mécontentement étaient couramment enregistrées à l'occasion des enquêtes préalables à l'autorisation d'installation, enquêtes dites de commodo et incommodo. Celles-ci étaient prolongées par des plaintes et des pétitions, les riverains devenant les auxiliaires de l'administration pour signaler les manquements de leurs voisins industriels ${ }^{16}$. Les déversements de matières, les rejets d'acide, le bruit et les trépidations, les risques d'incendie ou d'épidémie et une multitude infinie d'autres débordements viennent s'ajouter aux nuisances plus anciennes au fil de l'intensification industrielle.

Les temps qui précédèrent les mouvements écologistes au $20^{\mathrm{e}}$ siècle en France ne forment donc pas un paysage paisible et serein, sans pollutions ni contestations. Le pays était en effervescence industrielle au cœur même de la ville. Les confrontations étaient constantes entre intérêts contradictoires, industriels, artisans, exploitants avides d'exercer sans entraves leurs activités débordantes. Incendies criminels, menaces, agressions, bris de matériel, mise à sac pourraient bien avoir été plus nombreux que ne le laisse paraître la discrétion de l'histoire des impacts industriels. Quelques cas de troubles, d'attaques de sites commencent à apparaître ici et là. L'attentat au lance-roquette contre le chantier de la centrale de Creys-Malville en 1982, ou celui à la bombe contre une usine d'aluminium à Martigny en 1985 sont néanmoins tout à fait exceptionnels ${ }^{17}$. Les manifestations de violence ne sont en réalité pas les situations

${ }^{13}$ ( ) Pierre-Claude Reynard, «Public Order and Privilege : Eighteen-Century French Roots of Environmental Regulation», Technology and Culture, 43, 2002, p. 1-48; Morton J. Briggs, «Pollution in Poullaouen », Technology and Culture, 38, 1997, p. 635-654.

${ }^{14}$ ( ) Xavier Daumalin, «Industrie et environnement en Provence sous l'Empire et la Restauration », Rives méditerranéennes, 23, 2006, p. 27-46 ; Jean-Baptiste Fressoz, op. cit.

${ }^{15}$ ( ) Simon Edelblutte, «"Les montagnes qui poussent” ou l'impact des rejets salins des soudières de l'agglomération nancéienne », Revue de géographie de Lyon, 74, 1999, p. 243-251.

${ }^{16}$ ( ) Alain Corbin, «L'opinion et la politique face aux nuisances industrielles dans la ville préhausmanienne », Histoire, économie et société, 2, 1983, p. 111-118; Jean-Pierre Baud, « Le voisin protecteur de l'environnement », Revue juridique de l'environnement, 1, 1978, p. 15-33.

${ }^{17}$ ( ) Respectivement Chaïm Nissim, L'Amour et le Monstre : roquettes contre Creys-Malville, Paris, Favre, 2004 ; Dominique Ruch, «Une étonnante longévité : l'histoire d'une usine d'aluminium à Martigny », Cahiers d'histoire de l'aluminium, 42-43, 2009, p. 85-107. 
les plus répandues. Le principal registre visible de la conflictualité fut le recours légal au tribunal civil par des propriétaires fonciers ou des exploitants agricoles pour l'obtention de dédommagements.

En dehors de ce registre, les études spécifiques concernant les répertoires d'action des riverains protestataires sont rares. Une histoire des débordements et de leurs conflits pourrait ainsi explorer la palette des attitudes locales, au plus près des acteurs, voire des subalternes pour débusquer les stratégies de résistance mobilisées par les dominés. Elle pourrait considérer qu'ils n'ont pas nécessairement incorporé sans agir les conséquences de la production industrielle ${ }^{18}$. La reprise des travaux sur les résistances populaires à la mécanisation, sur les modes de l'action collective et individuelle autour du bris de machine aux $19^{\mathrm{e}}$ et $20^{\mathrm{e}}$ siècles invite par exemple à repenser les grammaires de la contestation en partant des dispositifs même de la production ${ }^{19}$.

\section{Des acteurs oubliés parmi les subalternes}

La plupart des études sur les conflits environnementaux ne porte en effet que sur une période débutant avec les années $1970^{20}$. La contestation y est souvent présentée comme la rupture du consensus sur les bienfaits de la société industrielle. Elle serait le produit de la dette héritée de l'industrialisation ${ }^{21}$. Les protestations ne se seraient en quelque sorte exprimées qu'avec l'apogée de la mobilisation environnementale dans le prolongement des mouvements écologistes $^{22}$. De même qu'une histoire de l'environnement n'aurait pas de sens avant son institutionnalisation et son existence politique, celle des conflits environnementaux serait tout autant sans objet ni combattant avant une date récente. À quelques exceptions près, les populations ne protestaient pas ou bien peu. Les préoccupations immédiates, urgentes,

${ }^{18}$ ( ) Gayatri Chakravorty Spivak, Les Subalternes peuvent-elles parler ?, Paris, Éd. Amsterdam, 2009 ; James C. Scott, La Domination et les arts de la résistance : fragments du discours subalterne, Paris, Éd. Amsterdam, 2008.

${ }^{19}$ ( ) François Jarrige, Au temps des «tueuses de bras»: les bris de machines à l'aube de l'ère industrielle, Rennes, Presses universitaires de Rennes, 2009.

${ }^{20}$ ( ) Olivier Filleule, « France », in Christopher Rootes (dir.), Environmental Protest in Western Europe, Oxford, Oxford University Press, 2003, p. 59-79; Graeme Hayes, Environmental Protest and the State in France, Palgrave Macmillan, 2002 ; Bruno Charlier, «La Défense de l'environnement : entre espace et territoire. Géographie des conflits environnementaux depuis 1974 », thèse de géographie, Université de Pau, 1999.

${ }^{21}$ ( ) Un modèle du genre : André Gortz, Écologie et Politique, Paris, Éd. du Seuil, 1978.

${ }^{22}$ ( ) Christopher Rootes (dir.), Environmental Movements : Local, National and Global, lieu?, Routledge, 1999, p. 1-12 ; René Pierre Chilbret, «Les Associations écologistes en France et en Allemagne : une analyse culturelle de la mobilisation collective », thèse de sociologie ?, Université Paris-I, 1991. 
quotidiennes s'imposaient trop impérieusement pour laisser place à la volonté d'agir. Il n'était pas question non plus d'attribuer à la nature une valeur autre qu'utilitaire, de considérer l'environnement dans le sens que nous lui accordons aujourd'hui. Prévalait plutôt la volonté de rationaliser les usages de la nature par les sciences et les techniques, d'être partie prenante du progrès industriel en marche ${ }^{23}$.

Un peu rapide, l'observation conduirait à considérer que la contestation environnementale serait un luxe réservé aux classes privilégiées. Elle serait une affaire d'opulents ou, dans sa version NIMBY (not in my backyard), une mobilisation de cyniques égoïstes pour la protection de leur environnement local au détriment de l'intérêt général ${ }^{24}$. À son opposé, l'autochtone, le profane, l'ouvrier, le subalterne formeraient une classe d'opprimés réputée inconsciente, incompétente et généralement irrationnelle.

Rien n'est plus illusoire. Preuve en est la perpétuation des luttes pour la ressource, le territoire et la santé, dans lesquelles des populations parmi les plus misérables s'engagent face à des multinationales adaptant leurs débordements aux conditions politiques et sociales d'exploitation de la ressource et de la main-d'œuvre locales. Souvent décrites comme des victimes passives de leur sort, les populations concernées s'avèrent parfois être aussi les actrices de leur combat. Les travaux sur l'injustice environnementale ont amplement démontré que si les plus défavorisés souffrent de façon disproportionnée des débordements qui leur sont infligés, ils ne restent ni démunis ni sans conscience ${ }^{25}$. L'environnement doit dès lors être regardé comme un lieu spécifique de la négociation pour d'autres motifs que la seule survie $^{26}$.

Envisager une histoire des débordements industriels et de leurs conflits nécessite dès lors d'expliciter le sens attribué par les acteurs concernés à ce qui fait l'objet de la contestation. Loin d'être un prétexte, l'environnement est bien l'une des raisons profondes de la mobilisation, à condition toutefois de redéfinir son statut et ses enjeux dans des termes

${ }^{23}$ ( ) Samuel P. Hays, Conservation and the Gospel of Efficiency: The progressive Conservation Mouvement, 1890-1920, Cambridge, Havard University Press, 1999.

${ }^{24}$ ( ) Jean-Marc Dziedzicki, «Au-delà du Nimby : le conflit d'aménagement, expression de multiples revendications », in Patrice Mélé, Corinne Larrue et Muriel Rosemberg (dir.), Conflits et territoires, Tours, Éd. de la MSH, 2004, p. 35-64.

${ }^{25}$ ( ) Timothy Doyle, Environmental Movements in Minority and Majority Worlds: A Global Perspective, New Brunswick, Rutgers University Press, 2005 ; Juan Martinez Alier, The Environmentalism of the Poor : A Study of Ecological Conflicts and Valuation, lieu ?, Edward Elgar Publishing, 2002.

${ }^{26}$ ( ) Geneviève Massard-Guilbaud et Richard Rodger (dir.), Environmental and Social Justice in the City : Historical Perspectives, Cambridge, White Horse Press, 2011. 
différents de ceux fixés par les promoteurs habituels de l'action ${ }^{27}$ : les conflits sont toujours la manifestation d'une rupture d'équilibre entre des rapports de domination. Il n'y a pas a priori de raison de dissocier leurs motivations, aujourd'hui comme dans le passé, de leurs enjeux environnementaux, que ce soit parmi des indigènes dans les colonies ou parmi des classes populaires françaises.

Dans cette perspective, les études sur les subalternes, et dans une certaine mesure les études postcoloniales, invitent à reconsidérer une sphère autonome de l'action politique dans l'univers des dominés, à voir opérer des mouvements d'opposition là où sont généralement vus des rivalités tribales ou ethniques, des confrontations entre des experts et des profanes irrationnels. Dans tous les cas, les termes de la protestation sont contextuels et historiquement situés. Les conflits s'ancrent ici dans une histoire politique et sociale heurtée, plongent leurs racines dans la rudesse d'une histoire industrielle au $19^{\mathrm{e}}$ siècle, là-bas dans un passé de la violence coloniale. Les conflits politiques, sociaux, économiques, ethniques, religieux, identitaires et territoriaux sont ainsi réinterprétés au prisme des enjeux environnementaux, dont les soubassements sont l'exploitation de la ressource et de la main-d'œuvre autochtones, liés à la gestion locale d'intérêts globalisés.

Environnement, débordement et conflit sont depuis longtemps intriqués. Cette permanence contredit l'image trop lisse d'une conscience collective surgissant au $20^{\mathrm{e}}$ siècle des ténèbres industrielles. Cette façon de cantonner l'environnement à une prise de conscience récente n'aide pas en réalité à concevoir tout le poids du passé sur les attitudes et les comportements actuels. Ignorer les fondements historiques de ces débordements et de leurs conflits ne permet pas de comprendre quelques paradoxes troublants. Parmi d'autres, celui qui fait apparaître sur le temps plus ou moins long des décalages entre l'histoire des savoirs et des innovations techniques, l'histoire des contestations environnementales et des mobilisations collectives, l'histoire de l'intervention publique ou de la législation. Le silence des riverains baignant dans les pollutions à une époque contraste par exemple avec la virulence de leurs protestations au moment même où la pollution en question semble ne plus être ou presque plus. Un dernier exemple précisera l'idée.

${ }^{27}$ ( ) David Schlosberg, Environmental Justice and the New Pluralism: The Challenge of Difference for Environmentalism, Oxford, Oxford University Press, 1999 ; Claudette Lafaye et Laurent Thévenot, «Une justification écologique ? Conflits dans l'aménagement de la nature », Revue française de sociologie, 34, 1993, p. 495-524. 


\section{Tonnes de chrome et motte de terre}

Une motte de terre noire sur la chaussée de Moulins-sur-Céphons provoque, au printemps 2008, la colère des riverains qui forment une association pour la défense de la qualité de vie dans les vallées de la Céphons et du Nahon dans l'Indre. Durant plusieurs jours, ces quelques centimètres cubes de boue ont défrayé la chronique locale, se sont exposés sur les écrans des médias audiovisuels et dans la presse. Qu'est-ce qui pouvait bien motiver la mobilisation des populations alentour? Elles protestaient contre les risques pour la santé et l'environnement que ferait courir la traversée de leur localité par ces matières contaminées en provenance de la rivière voisine. Ces boues étaient en effet acheminées par des bennes non étanches vers une décharge agricole locale, cette dépollution ayant été commandée suite aux débordements cumulés d'une industrie mégissière, elle-même héritière des tanneries médiévales ${ }^{28}$.

Cette industrie s'était surtout développée à Levroux au début du $19^{\mathrm{e}}$ siècle. Les procédés de fabrication donnaient alors lieu à d'importantes nuisances, mais aussi à une intense pollution du ruisseau local dans lequel les mégissiers déversaient leurs déchets. La population locale subissait les conséquences sanitaires d'un environnement délétère. Il était tel qu'en 1898 une épidémie de fièvre typhoïde sévit durant plusieurs mois. Les pollutions, notamment chimiques, s'aggravèrent dans les années 1960 avec une phase nouvelle d'industrialisation et l'usage de substances rejetées en grandes quantités dans les cours d'eaux, en particulier des bains de chrome, métal lourd toxique, cancérigène sous sa forme hexavalente à laquelle il est susceptible de parvenir lors de la manipulation des boues contaminées. Cette concentration de polluants avait fait disparaître depuis longtemps la faune aquatique. Après les années 1990, la période était devenue plus propice au traitement du site et à sa réaffectation, l'industrie ayant pour l'essentiel disparu sous sa forme initiale.

Exposée ainsi, la situation conflictuelle durant deux siècles de vie artisanale et industrielle peut bien susciter le sentiment d'une incompréhensible disproportion dans le traitement réservé à ces affaires. Une conclusion rapide pourrait même conduire à juger comme irrationnel le comportement des contestataires et à discréditer aujourd'hui leur prise de parole dans l'espace public.

Quelques décentrements sont pourtant nécessaires. En premier lieu, les enjeux ne peuvent se réduire ni à la qualification experte de la pollution par le chrome contenu dans une motte de terre, ni au lien tangible entre des quantités controversées de toxiques et leur perception par

\footnotetext{
${ }^{28}$ ( ) Armelle Querrien, «La mise en œuvre de l'énergie hydraulique en Berry : les moulins du bassin de la Céphons», in Le Village médiéval et son environnement: études offertes à Jean-Marie Pesez, Paris, Publications de la Sorbonne, 1998.
} 
des plaignants. La réalité biophysique des interactions entre des molécules et des organismes vivants n'est certes pas à ignorer, mais elle n'est pas suffisante pour saisir une dynamique de nature profondément politique, culturelle et sociale. L'environnement en question n'est pas un objet déjà là, assemblé et stable de toute éternité. Il est le produit d'une histoire, d'une projection des identités locales, d'une renégociation pour la qualification des espaces et pour l'attribution de fonctions aux territoires.

La motte de la discorde n'est ici qu'un élément parmi d'autres dans la trajectoire d'un conflit environnemental liés aux débordements cumulés des anciennes tanneries. Leur sédimentation locale a formé le terreau fertile sur lequel pouvait s'ériger la confrontation d'intérêts contradictoires. Se déploie autour de la motte de terre un dispositif dans lequel sont convoqués, entre autres, des instruments de mesure de la contamination par le chrome, des normes légales, mais également des industriels d'aujourd'hui et d'hier, des populations salariées ou résidentes alentour, des médecins et des hygiénistes, des représentants de l'autorité publique, des propriétaires fonciers, des poissons qui ont depuis longtemps disparu de la rivière... Ne faudrait-il pas dès lors prendre au sérieux nombre de ces acteurs et ne pas leur imputer des intentions forcément cachées, mais entendre ce qu'ils disent et observer ce qu'ils font, afin de percevoir, au travers d'une histoire singulière, la manière dont ils se rattachent à une politique plus large de l'environnement?

\section{Une histoire du conflit environnemental}

Au premier abord, le conflit environnemental peut donc être regardé comme la manifestation des tensions entre des acteurs sociaux dont le motif est la confrontation des fonctions attribuées à un environnement donné. Ce dernier est un espace en partage. Des intérêts divergents en négocient les usages. Il y a donc conflit quand les fonctions attribuées à un environnement donné sont contrariées. Elles le sont parce qu'elles portent atteinte à l'intégrité d'individus ou à celle de catégories de populations, à celle d'êtres vivants ou à des écosystèmes. Classiquement, ces intérêts contradictoires se disputent l'appropriation de ressources, d'espaces et de territoires. Ils sont susceptibles de porter atteinte à des valeurs, à des identités, à des rapports à la nature projetés dans cet environnement.

Cette façon d'observer le conflit environnemental est-elle spécifique à l'histoire du temps présent? Ne s'avère-t-elle pas tout aussi pertinente pour interpréter les affrontements du passé, le conflit environnemental devenant lui-même un outil pour l'histoire de ces confrontations que suscitent l'altération des milieux, l'usage de la ressource, le paysage, la santé, le territoire, le risque non consenti ? Le conflit environnemental ne peut dans ce cas pas 
être conceptualisé comme une opposition portant sur un problème prédéfini et sans équivoque, avec des acteurs dont les points de vue et les intérêts seraient seulement en concurrence. Il doit plutôt être vu comme une négociation complexe et permanente pour imposer une définition et une signification au problème environnemental ${ }^{29}$.

Le terme «environnement 》 doit être réévalué, d'une manière compréhensive, intégrant tout ce que peuvent comporter en nature et en culture des espaces appropriés ${ }^{30}$. Il est question de structures humaines autant faites de nature que d'organisations politique et sociale. Autrement dit, le terme «environnement » ne se réfère ni à un état de nature auquel serait attribuée une valeur intrinsèque, ni à cette catégorie spécifique de l'action ou de la pensée politique, mais plus largement à l'ensemble de ces éléments hybrides, inscrits dans des histoires et constituant des territoires dont les statuts et les qualifications font l'objet, à un instant donné, d'une renégociation, d'une réaffirmation par le conflit. Ce qui suppose de devoir situer son improbable définition au cœur même des transactions pour la délimitation des frontières entre espaces publics et privés, entre logiques techniques et mondes du vivant, entre représentations et réalités vécues.

Cette approche considère l'environnement comme une réalité nécessairement plurielle. Elle tient compte de trois composantes irréductibles qui ne peuvent jamais être totalement ignorées, même si elles peuvent être isolées pour les besoins de l'enquête historique : la géographie des territoires, siège de la conflictualité entre local et global ; l'histoire, ou plutôt les histoires dans lesquelles s'inscrivent les termes du conflit entre passé et devenir; enfin, une composante hybride, entre nature et culture.

Une histoire des débordements industriels et de leurs conflits commande donc de porter une attention particulière aux lieux, ainsi qu'aux processus concurrents d'appropriation de territoires dont les fonctions initiales sont contrariées par ce qui déborde. La démarche implique de s'intéresser aux négociations en vue de l'attribution de ce statut de débordement aux matières réelles ou intangibles concernées. Ce sont ces négociations qu'il s'agit de restituer, négociations menées dans les arènes publiques de débat, la cité administrative et politique, les enceintes judiciaires, les sphères de production de la norme, les domaines spécifiques de l'intervention publique, les lieux de la prise de décision, ceux de la science et de l'expertise, et autant d'autres espaces de la controverse et de la légitimation.

L'interprétation des conflits du passé, à partir de la notion de débordement, invite in fine à

${ }^{29}$ ( ) Maarten A. Hajer, The Politics of Environmental Discourse : Ecological Modernization and the Policy Process, Oxford, Clarendon Press, 1997, p. 14. 
une articulation entre une histoire de la conflictualité environnementale et une histoire politique de l'émergence de l'environnement. Elle permet d'éviter l'écueil qui consisterait à laisser s'effacer des acteurs et des enjeux derrière les catastrophes majeures au retentissement national, voire international. Le bruit de fond formé par la multitude des conflits locaux durant l'industrialisation résonne toujours aujourd'hui dans la cité. L'histoire de la conflictualité éclairerait ainsi sans doute le passage d'une société industrielle productrice de débordements à une société malade de leurs conséquences. L'image d'un tournant environnemental de la société industrielle qui aurait désormais autorisé les conflits peut alors s'apprécier au prisme d'une histoire longue des débordements eux-mêmes.

\section{Nuancer la perception du tournant environnemental}

Le traumatisme des restructurations industrielles a incontestablement transformé les rapports qu'entretiennent les Français avec leur industrie, une activité économique qu'ils n'aimeraient plus. Le contraste est saisissant entre l'exaltation d'une industrie triomphante au début du siècle dernier, et sa stigmatisation sept ou huit décennies plus tard. Les rejets industriels ontils finalement conduit au rejet de l'industrie? La saturation des milieux par les polluants, l'écologie et le militantisme pour la conservation d'une nature originelle, la prégnance dans les esprits des catastrophes ont-ils favorisé la mobilisation contre les pollutions et les risques ? Doit-on considérer que l'explosion de Feyzin en 1966, ou le naufrage du Torrey Canyon l'année suivante inaugurent l'ère des grandes catastrophes environnementales et celle d'une prise de conscience collective conséquente en France?

Des événements majeurs de cet ordre ont sans aucun doute contribué à inscrire à l'agenda politique et médiatique les questions d'environnement. L'explosion de la poudrerie de Grenelle (1794), la catastrophe de Courrière (1906) et la destruction de l'usine d'AZF (2001) ont tellement marqué l'imaginaire social qu'elles eurent un rôle déterminant dans l'élaboration de la législation sur les établissements classés. L'explosion de la poudrerie de Grenelle est ainsi devenue, dans les textes officiels, l'événement à l'origine d'une histoire de la gestion du risque industriel en France. Le lien n'est pourtant pas évident. Ce qui l'est plus en revanche, c'est la nécessité d'inscrire aujourd'hui l'action des pouvoirs publics dans une généalogie, une histoire, dont la fin, déjà connue, est celle de la conscience des problèmes et des bonnes pratiques de gouvernance. Elle invite à la quête d'une autre rationalité pour un nouvel ordre économique et social, fixant les cadres de l'action publique contemporaine. L'extension de l'environnement à tous les aspects de l'activité humaine, tout comme les

\footnotetext{
${ }^{30}$ ( ) Marc Mormont, « Conflit et territorialisation », Géographie, économie, société, 3, 2006, p. 299-318.
} 
injonctions au développement durable, commandent un avenir désormais responsable, qui serait de stabiliser la définition gestionnaire des possibles entre loi du marché et fin des utopies. L'environnement s'ancre assurément dans une évolution au $20^{\mathrm{e}}$ siècle des sensibilités et du rapport à la nature, de la législation et de la régulation des activités dangereuses, ponctuée par les accidents, les pollutions à grande échelle et les intoxications massives. En quelque sorte, la revendication pour un environnement sain et sûr est le signe que la société industrielle aurait atteint sa pleine maturité et le stade ultime de sa modernité.

Cette proposition doit être néanmoins confrontée à l'histoire, sur le temps long, des débordements et de leurs conflits. Il s'agit alors de réintroduire des acteurs et des enjeux là où ils ont eu tendance à disparaître derrière la vision d'un tournant environnemental de la société industrielle. Une révision des échelles s'impose. La démarche implique de distinguer plusieurs niveaux d'analyse, de temps et d'espace. En observant ce qui se joue au niveau local, en s'éloignant du grand récit de la prise de conscience écologiste, la pluralité des motifs devient perceptible, ainsi que la complexité des rapports des acteurs à la ressource, au territoire, à l'espace et au paysage, au milieu. L'attention portée aux débordements et à leurs territoires permet en particulier de percevoir comment est advenu par le conflit ce qui s'inscrit désormais sous l'égide du terme environnement. Cette histoire enfin s'intéresse aux cadres et aux formes de la mobilisation, dont la nature évolue avec la législation et les modalités de l'intervention publique. Elle insiste sur les dynamiques de leur territorialisation. Elle incite à décentrer le regard pour faire émerger des versions différentes du conflit environnemental et à finalement considérer qu'il y a toujours pluralité des ordres de réalité des débordements aux sources de ces conflits.

Michel Letté, Conservatoire national des arts et métiers (CNAM), Centre d'histoire des techniques et de l'environnement (CHTE), 75003, Paris, France.

Maître de conférences au CNAM de Paris, Michel Letté enseigne dans le cadre du master d'histoire des techniques et de la chaire Unesco « Mémoire des métiers vivants » du CHTE. Il est responsable de l'équipe «Environnements, techniques, conflits » et dirige le programme «Débordements industriels dans la cité : études de conflits et d'interventions publiques aux $19^{\mathrm{e}}$ et $20^{\mathrm{e}}$ siècles ». (michel.lette@ cnam.fr) 